\title{
Transtorno do espectro autista: o impacto na dinâmica familiar e as habilidades no cuidado
}

\author{
Autism spectrum disorder: the impact on family dynamics and care skills
}

Trastorno del espectro autista: el impacto en la dinámica familiar y las habilidades de cuidado

Valéria Gomes Marques ${ }^{1 *}$, Nívia Katiele Maria dos Santos², Thamires de Souza Marques ${ }^{1}$, Maria Clara Almondes de Azevedo ${ }^{1}$, Márcio Moreira Cajahiba Júnior ${ }^{1}$, Carolina Borba Freire ${ }^{1}$, Beatriz Santos Farias ${ }^{1}$, Sâmia Cidreira Sufi ${ }^{1}$, Laura Virgínia Prazeres Cotrim ${ }^{1}$, Michela Macedo Lima Costa ${ }^{1}$.

\section{RESUMO}

Objetivo: Entender o Transtorno do Espectro Autista (TEA) no que tange as suas características comportamentais, o impacto disso na dinâmica familiar, além de identificar algumas habilidades no cuidado. Revisão bibliográfica: As manifestações comportamentais do TEA se apresentam precocemente. Dentre eles, tem-se irregularidade e atraso no controle e desenvolvimento motor; dificuldade para reconhecer emoções, apresentando um afeto negativo; sensibilidade diminuída a recompensas sociais; e dificuldade para manter sua atenção. Em razão disso, toda a rotina e dinamicidade do núcleo familiar acaba sofrendo alterações e, com isso, altos níveis de estresse e ansiedade podem estar presentes entre os cuidadores, sobretudo, nas mães. Assim, incentivar o desenvolvimento de habilidades intervencionistas pelos cuidadores através de atividades que incentivem a comunicação e socialização, por exemplo, é fundamental, a fim de possibilitar um melhor acolhimento ao indivíduo. Considerações finais: O TEA ainda hoje é muito estigmatizado pela sociedade. Isso, infelizmente, se perpetua, dentre outras razões, por carência de pesquisas com embasamento teórico-científico que possa compreender melhor as suas características, além das próprias ideologias civis, resultando, dentre outros fatores, em comportamentos superprotetores.

Palavras-chave: Autismo, Transtorno do espectro autista, Dinâmica familiar.

\begin{abstract}
Objective: To understand Autism Spectrum Disorder (ASD) in terms of its behavioral characteristics, its impact on family dynamics, in addition to identifying some skills in care. Bibliographic review: The behavioral manifestations of ASD are presented early. Among them, there is irregularity and delay in motor control and development; difficulty recognizing emotions, showing negative affect; decreased sensitivity to social rewards; and difficulty keeping your attention. As a result, the entire routine and dynamics of the family unit ends up undergoing changes and, with this, high levels of stress and anxiety may be present among caregivers, especially in mothers. Thus, encouraging the development of interventionist skills by caregivers through activities that encourage communication and socialization, for example, is essential, in order to enable a better reception for the individual. Final considerations: The TEA is still very stigmatized by society today. This, unfortunately, is perpetuated, among other reasons, by the lack of research with a theoretical-scientific basis that can better understand its characteristics, in addition to the civil ideologies themselves, resulting, among other factors, in overprotective behavior.
\end{abstract}

Keywords: Autism, Autistic spectrum disorder, Family dynamics.

\footnotetext{
${ }^{1}$ Faculdade Santo Agostinho (FASA), Vitória da Conquista - BA. *E-mail: valeria.fasavic@gmail.com

2 Universidade do Estado da Bahia (UNEB), Salvador - BA.
} 


\section{RESUMEN}

Objetivo: Comprender el Trastorno del Espectro Autista (TEA) en cuanto a sus características conductuales, su impacto en la dinámica familiar, además de identificar algunas habilidades en el cuidado. Revisión bibliográfica: las manifestaciones conductuales del TEA se presentan temprano. Entre ellos, hay irregularidades y retrasos en el control y desarrollo motor; dificultad para reconocer emociones, mostrando afecto negativo; disminución de la sensibilidad a las recompensas sociales; y dificultad para mantener su atención. Como resultado, toda la rutina y dinámica de la unidad familiar termina sufriendo cambios y, con ello, pueden presentarse altos niveles de estrés y ansiedad entre los cuidadores, especialmente en las madres. Así, es fundamental fomentar el desarrollo de habilidades intervencionistas por parte de los cuidadores a través de actividades que fomenten la comunicación y la socialización, por ejemplo, para posibilitar una mejor recepción por parte del individuo. Consideraciones finales: La TEA todavía está muy estigmatizada por la sociedad en la actualidad. Esto, lamentablemente, se perpetúa, entre otras razones, por la falta de investigaciones con base teórico-científica que permitan comprender mejor sus características, además de las propias ideologías civiles, resultando, entre otros factores, en conductas sobreprotectoras.

Palabras clave: Autismo, Trastorno del espectro autista, Dinámica familiar.

\section{INTRODUÇÃO}

O Transtorno do Espectro Autista (TEA), possui um caráter heterogêneo, devido a sua etiologia multifatorial, mas, em geral, é determinado por uma deficiência na comunicação e interação social, e por um comprometimento neurocomportamental, com interesses e atividades repetitivas (STEYER S, et al., 2018).

Estudos epidemiológicos desenvolvidos pela Gobal Burden of Disease, em 2010, mostraram que o autismo é mais prevalente em homens do que em mulheres, apresentando uma relação de proporcionalidade estimada de 4:1. Ademais, é uma condição altamente complexa, cuja etiologia é categorizada em dois grupos: forma primária, também conhecida como essencial, de origem multifatorial; ou ainda, na forma secundária ou sindrômica, que possui uma causa identificada (ALMEIDA SSA, et al., 2018).

A classificação do TEA em essencial e sindrômica tem ainda como princípio a variação morfológica. Na sua forma essencial, os indivíduos apresentam menor chance de ter uma síndrome genética e, consequentemente, menos dimorfismos, deficiência intelectual e comorbidades associadas. Em contrapartida, possui mais história familiar psiquiátrica e maior diferença entre os sexos $(4 \mathrm{H}: 1 \mathrm{M})$. Já o sindrômico é o inverso, tem maior chance de ter uma síndrome genética, dimorfismos, ocorrência de deficiência intelectual, epilepsia e malformações. Porém, menos história familiar (evento de novo) e menor diferença entre os sexos (1H:1M). Além disso, apresentam uma subcategoria que engloba os aspectos genéticos e ambientais (TOMASI MC, et al., 2018).

Quanto aos padrões genéticos, apresentam três vertentes: a primeira corresponde as alterações em pares de base específicos, conhecidas como "variantes de nucleotídeos únicos"; a segunda refere-se as "variantes do número de cópias", que nada mais são do que deleções ou duplicações de múltiplos pares de bases; e, por fim, a terceira que relata o envolvimento de algumas síndromes genéticas, como a síndrome do $\mathrm{X}$ frágil, síndrome de Rett e a esclerose tuberosa (WENGER TL, et al., 2016).

Em relação aos fatores ambientais, foi identificada uma forte correlação entre o autismo e as condições do recém-nascido, ou seja, prematuridade, baixo peso ao nascer, intoxicações fetais, carência de vitamina $D$ e ácido fólico. Por outro lado, o risco aumenta-se também quando as mães desses indivíduos têm infecções e exposição de ácido valproico durante a gestação, causando uma interação direta com o genoma celular (HADJKACEM I, et al., 2016).

Quanto as pesquisas de padrões neuroanatômicos envolvendo o TEA, começaram no final da década de 80, através de estudos post-mortem, onde foram descritas alterações em algumas regiões encefálicas, dentre elas, destacam-se o sistema límbico e os circuitos cerebelares. Na época, o cerebelo foi o mais bem estudado, 
onde através da análise de exame de imagem, especificamente, por meio da Ressonância Magnética (RM), foi possível identificar uma hipoplasia nos lóbulos vermianos VI e VII em um grupo de pacientes com autismo (OLIVEIRA CRA e SOUZA JC, 2021).

A amígdala é uma estrutura cerebral complexa que no autismo, apresenta alterações anatômicas no seu tamanho, apontando uma forte correlação entre o seu volume e a gravidade do quadro clínico, o que configura uma dificuldade no reconhecimento e nas respostas emocionais, e uma tendência à agressividade e medo (VILANI MR e PORT IF, 2018).

Já o hipocampo, estrutura adjacente à amigdala, localizado nos lobos temporais cerebrais. Em crianças autistas, com ou sem atraso mental, apresentam assim como a amígdala, uma alteração no volume do hipocampo direito quando comparado aos não autistas, o que pode sugerir uma excelente memória de longo prazo, sobretudo para fatos e estatísticas (LIMA RC, 2020).

O giro do cíngulo, localizado logo abaixo do corpo caloso é formado por um aglomerado de fibras que fazem a comunicação entre o sistema límbico e o córtex. Em alguns estudos sobre o TEA foi-se observada tanto uma redução do seu volume na porção anterior direita, especificamente na área 24 de Brodmann, relacionado à motricidade, quanto uma hipoativação dessa região, o que porque presumir uma dificuldade para os autistas em focalizar a sua atenção para eventos emocionalmente significantes e também para fazer uma associação de memórias a odores e dor (SIQUEIRA S, 2016).

O corpo caloso, por sua vez, é responsável por facilitar a comunicação entre os dois hemisférios cerebrais e também se encontra alterado no TEA com uma diminuição no seu tamanho, sobretudo, no terço caudal. Já o corpo mamilar está intimamente relacionado ao hipotálamo, e no autismo ele se encontra comprometido, havendo uma hipoativação dessa área, o que confere prejuízo da capacidade de aprendizagem espacial (OLIVEIRA CRA e SOUZA JC, 2021).

Assim, esse constructo teve como objetivo entender o TEA no que tange as suas características comportamentais, o impacto disso na dinâmica familiar, além de identificar algumas habilidades no cuidado.

\section{REVISÃO BIBLIOGRÁFICA}

\section{Traços comportamentais do autismo}

As manifestações comportamentais do TEA se apresentam precocemente, mas a sua trajetória inicial não é uniforme. Isso significa que em algumas crianças, os seus sintomas podem ser aparentes logo após o nascimento, porém tende a ser mais frequentemente identificados entre os 12 e 24 meses de idade, apesar de que o diagnóstico ocorre, em geral, entre 4 ou 5 anos (PINTO RNM, et al., 2016).

No entanto, ainda vale ressaltar que essas características clínicas do transtorno autístico dependem não apenas da idade cronológica, mas também variam muito dependendo da gravidade da condição pautado na funcionalidade, de acordo com o Manual de Diagnósticos e Estatísticas de Distúrbios Mentais (DSM-5), ou ainda, por conta dos em níveis de deficiência intelectual e linguagem funcional, como aborda o CID-11; daí o uso do termo espectro (FERNANDES CS, et al., 2020).

Estudos de caráter científico já comprovaram a forte correlação entre uma intervenção precoce e a significativa melhoria no funcionamento cognitivo e adaptativo da criança autista. Isso se deve pelo fato de que é exatamente dos períodos iniciais da vida que cérebro é altamente plástico e maleável, o que torna mais permissivo moldar os padrões de comportamento (ZWAIGENBAUM L, et al., 2015).

Diante disso, é crucial conhecer e identificar quais são os primeiros sinais potencialmente importantes para o autismo logo no primeiro ano de vida. Dentre eles, tem-se irregularidade e atraso no controle e desenvolvimento motor; dificuldade para reconhecer emoções, apresentando um afeto negativo; sensibilidade diminuída a recompensas sociais; e dificuldade para manter sua atenção (ANAGNOSTOU E, et al., 2014).

Apesar da complexidade no reconhecimento de comportamentos típicos do autismo logo após o nascimento, ainda assim, é possível identificar sinais de alerta. No lactante, pode haver um atraso para 
expressar o sorriso social, apresenta um olhar não sustentado ou ausente, com pouco interesse pela face humana, em contrapartida, há uma grande preferência por objetos que sorriem para eles e que movimentam o corpinho. Além disso, não se sentem confortáveis quando colocados no colo, demonstrando até mesmo certa irritabilidade, preferindo dormir sozinhos no berço e demonstram indiferença quando estão distantes dos pais (SOCIEDADE BRASILEIRA DE PEDIATRIA, 2017).

Com o avançar da idade, já é possível identificar os traços do autismo com mais precisão, pois eles tornam mais evidentes. Nesse caso, entre 12 meses a 16 meses pode verificar a ausência de balbucio, principalmente, de sílabas com consoantes, não apresenta uma comunicação gestual, ou seja, a criança não aponta para um objeto desejado nem olha para o indicador de outra pessoa, ela pode, inclusive, apresentar dificuldade para se comunicar e na fala não inclui palavras simples, além de "mamã" e "papá" (MANSUR OMFC, et al., 2017).

A partir dos 18 até os 24 meses, já percebe tanto atraso de linguagem verbal ou não-verbal quanto no contato social, interesses por comportamentos repetitivos e estereotipias (MANSUR OMFC, et al., 2017). Na alimentação, por exemplo, as crianças autistas podem apresentar recusa a depender de qualquer mudança seja na textura, consistência ou cor da comida ou até mesmo quanto a sua disposição no prato (MORAES LS, et al., 2021).

Entre 24 a 36 meses podem apresentar desinteresse para descrever fatos do seu cotidiano, apresentando repetição de falas de outras pessoas e, geralmente, não costumam imaginar cenas ou histórias em suas brincadeiras e quando fazem é com pouca criatividade, sendo bastante repetitivos. Além disso, têm predisposição por comportamentos antissociais e quando há algum tipo de interação social, normalmente, sentem dificuldade para entender as gesticulações e emoções dos demais envolvidos em uma brincadeira, por exemplo (MANSUR OMFC, et al., 2017).

Com o avançar da idade, alguns autistas podem desenvolver comportamentos incomuns que indicam um certo grau de severidade da sua condição. Dentre elas, podem apresentar movimentos motores estereotipados (flapping de mãos); ações atípicas repetitivas (demonstrar obsessão por determinados objetos em movimento, como ventiladores); dissimetrias na motricidade (maior movimentação dos membros de um lado do corpo) (MARQUES DF e BOSA CA, 2015).

As manifestações sensoriais atípicas, como o hábito de cheirar e/ou lamber objetos, alta sensibilidade a determinados sons, como secador de cabelos, insistência visual e tátil por objetos de brilham e/ou que apresentam determinada textura. Por fim, tem muita tendência a rotinas ritualizadas e rígidas e podem, gradual ou subitamente, deixar de falar e perder certas habilidades sociais já adquiridas por volta dos 12 aos 24 meses (POSAR A e VISCONTI P, 2018).

\section{Transtorno autístico e dinâmica familiar}

Receber o diagnóstico de TEA não impacta apenas o indivíduo em si, mas toda a sua família, despertando diferentes reações, que perpassam a aceitação ou negação, além da preocupação, sofrimento, culpa e medo do imprevisível. Isso, portanto, requer inúmeras descobertas, planejamentos e cuidados por parte dos integrantes da família, na tentativa de facilitar a adaptação e sensibilizar o acolhimento da pessoa com autismo (HOFZMANN RR, et al., 2019).

Em geral, a percepção de alterações no desenvolvimento biopsicossocial das pessoas com TEA são notadas pelas mães logo nas fases iniciais das suas vidas, buscando ajuda profissional, normalmente, na atenção primária. Isso faz com que elas se tornam, posteriormente, as principais cuidadoras informais, dedicando-se integralmente seu dia ao filho (PINTO AS e CONSTANTINIDIS TC, 2020).

Toda a rotina e dinamicidade do núcleo familiar acaba sofrendo alterações e, com isso, altos níveis de estresse e ansiedade podem estar presentes entre os cuidadores, sobretudo, nas mães, em razão da sobrecarga, o que aumentam as chances de desencadear transtornos de humor, principalmente a depressão, e ansiedade. Ademais, dor de cabeça, fadiga, tensão e dor muscular, podem também manifestar como sintomas físicos (DYKENS EM, et al., 2015). 
No entanto, esse redimensionamento das atividades cotidianas reflete também no empobrecimento das vivências sociais, com perdas de amizades; em conflitos conjugais, com aumento de discussões entre o casal que, por vezes, culmina em divórcio, justificados pela falta de colaboração aos cuidados do filho, no que diz respeito à divisão dos papeis de gênero; no comprometimento das carreiras profissionais, o que repercute no orçamento familiar, em razão dos gastos em tratamentos com psicólogos, neuropediatras e fonoaudiólogos (SANDIN S, et al., 2014).

Apesar do TEA provocar vários problemas no contexto familiar, é possível identificar, em contraste às dificuldades, uma maior proximidade na relação pais-filho, além de algumas habilidades desenvolvidas pelos indivíduos com autismo, desde facilidades na aprendizagem de outros idiomas, como também aptidão com cálculos, letras e eletroeletrônicos, por exemplo (HOFZMANN RR, et al., 2019).

\section{Acolhimento familiar no autismo}

Os traços comportamentais que caracterizam o TEA precisam ser levados em consideração no momento de promover tanto as mudanças no espaço físico familiar quanto no desenvolvimento das habilidades intervencionistas dos cuidadores, a fim de possibilitar um melhor acolhimento ao indivíduo. Nesse sentido, quanto as habilidades sociocomunicativas implementadas, é fundamental considerar, primeiramente, as habilidades do indivíduo e suas limitações. Portanto, aqueles que possuem um grande déficit na comunicação verbal, os cuidados podem lançar mão como comunicação alternativa, os sistemas de sinais (OLIVEIRA JJM, et al., 2020).

O Communication System by Exchange of Figures (PECS) é um exemplo de como uma criança com autismo que apresenta dificuldades na comunicação pode se adaptar no meio em que vive. Para isso, é necessário que os pais utilizem cards com determinadas imagens e velcro para indicar as ações desejadas dos seus filhos, sendo o equivalente à sua voz. Vale ressaltar que durante as fases iniciais, a figura/card funciona como um ticket que o indivíduo troca com o cuidador em função de receber algo. Já em fases mais avançadas, eles aprendem a formar frases, discriminar, usar atributos, responder perguntas e fazer comentários (SANTOS PA, et al., 2021).

Outras ações cruciais para estimular as habilidades sociocomunicativas são através de brincadeiras que permitem estimular o contato visual direto, como "esconder a face e dar um susto" e "fazer cócegas", ou ainda gesticulações com as mãos, como o "apontar", para permitir um direcionamento da atenção da criança para objetos específicos, a partir de comandos (OLIVEIRA JJM, et al., 2020).

Sabe-se que algumas das características do TEA são a rigidez com rotinas, comportamentos de distração e a falta de motivação. Nesse caso, pode ser desenvolvido um ensino estruturado adotando para isso o modelo Tratamento e Educação para Autistas e Crianças com Déficits Relacionados à Comunicação (TEACCH), seja em casa, na escola ou ainda no ambiente terapêutico, de modo a ensinar técnicas e métodos de educação especial aos pais, possibilitando a organização e sistematização de tarefas a serem realizadas, de modo que o aprendizado das crianças seja mais eficaz e fácil (BASTOS RP e SOUZA DC, 2019).

Esse método parte do princípio de abordar as áreas de maior domínio nas pessoas com TEA, ou seja, processamento visual, memorização de rotinas funcionais e interesses especiais e, a partir disso, elabora-se um planejamento extremamente flexível, procurando sempre atender as necessidades individuais e as diferentes limitações funcionais existentes, respeitando sempre a sua forma de pensar e aprender (BASTOS RP e SOUZA DC, 2019).

A sua estrutura básica é informar de maneira clara e objetiva todas tarefas que serão desenvolvidas durante o dia; manter o ambiente calmo e organizado, com delimitações evidentes de espaços para possibilitar que o indivíduo entenda o que acontecerá em cada segmento; afastar qualquer estímulo sensorial, como barulhos e luminosidade excessiva que possa causar desconforto e crises nesses indivíduos; e desenvolver atividades diárias que estejam ao seu alcance, respeitando os níveis de funcionalidade (CUNHA MCG, et al., 2019). 
No contexto de intervenções sensoriais, é necessário primariamente entender que no autismo existem os Transtornos de Processamento Sensorial (TPS), que nada mais são do que três eixos sensoriais que estão afetados e se caracterizam por: Transtornos Motores de Base Sensorial (TMBS), Transtornos de Discriminação Sensorial (TDS) e Transtornos de Modulação Sensorial (TMS) (MARTINS JS, et al., 2020).

O TMBS ainda se subdivide em outras duas categorias, como o transtorno postural, tendo dificuldade para manter o alinhamento postural, e a dispraxia que é a dificuldade para planejar e coordenar os movimentos do corpo. Isso, portanto, pode interferir diretamente o seu aprendizado motor grosso e/ou fino (MARTINS JS, et al., 2020).

O TDS, por sua vez, se caracteriza pelas dificuldade que pessoas com autismo têm em captar as semelhanças e diferenças entre estímulos, assim como distinguir a nível temporal e espacial, ou seja, sem o auxílio da visão, esses indivíduos não conseguem deduzir, apenas com o tato, de qual objeto se trata, como também sentem dificuldade para diferenciar visualmente, por exemplo, letras que são muito parecidas, como b e d ou p e q e, ainda, apresentam dificuldade para estimar o quanto de força é necessário aplicar para escrever, tendo, então, um comprometimento da propriocepção (MARTINS JS, et al., 2020).

O TMS que se caracteriza por dificuldade no ajuste e processamento das mensagens neurais que carregam informações sobre a intensidade, frequência, duração, complexidade e novidade de estímulos sensoriais, sendo categorizados em três grupos: os hiperresponsivos, os hiporresposivos e o de procura sensorial. Os indivíduos hiperresponsivos, diante de pouquíssimo estímulo atingem o limiar. Nesse sentindo, perante a exposição de barulhos de secador, fogos de artifícios, estouro de balões, brincadeiras que sujam, abraços frequentes e lugares muito cheios, por exemplo, acabam desenvolvendo um comportamento de recusa, além de estresse, ansiedade e nervosismo (SILVA ER, et al., 2016).

Já os hiporresponsivos, é o contrário, necessitam de muito estímulo para alcançar o limiar, tendo alta tolerância à dor, tocam em tudo e leva à boca, costumam ter comportamentos mais frequentes de autoagressão e necessitam estar em constante movimento, seja ele giratório ou balançando. Por fim, tem a procura sensorial que é definida como a procura por estímulos intensos, com maior duração e frequência. Nesse caso, esse indivíduo tem preferência por brincadeiras mais impulsivas, envolvendo colisões e quedas, além que preferir sons altos e movimentos rápidos (POSAR A e VISCONTI P, 2018).

Diante desse contexto, é importante que os pais sabiam identificar quais são os tipos de entradas sensoriais específicas que causam desconforto em determinado indivíduo, sendo o pré-requisito para reorganizar o ambiente em que ele vive e sua rotina diária para reduzir o máximo possível a ocorrência de crises. Nesse sentido, as intervenções se baseiam em dois tipos: a primeira está centrada no indivíduo com autismo, chamadas de "terapias de integração sensorial", enquanto que outra está voltada para o cuidador, ou seja, as "intervenções baseadas nos sensoriais" (CARDOSO NR e BLANCO MB, 2019).

Partindo desse princípio, se a criança sente um incômodo fora do normal ao tocar em determinadas texturas, os pais podem, sob orientação profissional, estimular esse contato aos poucos, usando para isso, algumas brincadeiras com massinha de modelar e ir, gradualmente, mudando para outras texturas, caso obtenha uma resposta positiva. Assim como, na hora banho, pode colocar esponjas/buchas de banho com diferentes texturas para tentar o estímulo sensorial também (VIANA KOFL e NASCIMENTO SS, 2021).

Além disso, estimular outras brincadeiras lúdicas como fechar os olhos e tentar tocar a ponta do nariz (ou outras partes do próprio rosto) ou ainda utilizar a prancha de equilíbrio é importante para o desenvolvimento do sistema proprioceptivo e também quanto à coordenação motora/equilíbrio. Usar balanço e gira-gira também podem colaborar para o desenvolvimento do equilíbrio corporal. Quanto as brincadeiras que podem ajudar o sistema auditivo, pode usar objetos que simulem um alto-falante ou ainda, instrumentos musicais. Já em relação ao sistema visual, pode valer de "brincar de achar com a lanterna", onde estimulará o indivíduo a achar o objeto no ambiente escuro com a lanterna (BRANT DVS, 2018).

Portanto, nessas situações, é fundamental que os pais avaliem as respostas comportamentais dos filhos, sem forçar a realização das atividades e se manter sempre paciente quando não obtiver sucesso nas primeiras tentativas e persistente na regularidade das ações (GOMES PTM, et al., 2015). 


\section{CONSIDERAÇÕES FINAIS}

O TEA ainda hoje é muito estigmatizado pela sociedade. Isso, infelizmente, se perpetua, dentre outras razões, por carência de pesquisas com embasamento teórico-científico que possa compreender melhor as suas características genéticas e neurocomportamentais. Ademais, têm-se as próprias ideologias civis de que essa condição, naturalmente, já coloca a pessoa com autismo em uma posição diferente e até inferior aos não autistas, de total dependência. Por essa razão, induz os próprios familiares a adotar comportamentos superprotetores, refletindo, por vezes, em estresse e ansiedade, por exemplo. Desse modo, considera-se de grande relevância o desenvolvimento dessa pesquisa, com a finalidade de buscar mostrar algumas medidas funcionais a serem aplicadas pelos integrantes da família, quanto habilidade no cuidado, a fim de criar um ambiente físico otimizado para a criança com autismo, facilitando no seu desenvolvimento cognitivo e social.

\section{REFERÊNCIAS}

1. ALMEIDA SSA, et al. Transtorno do Espectro Autista. Revista Residência Pediátrica, 2018; 8: 72-78.

2. ANAGNOSTOU E, et al. Autism spectrum disorder: advances in evidence-based practice. Canadian Medical Association Journal, 2014; 186(7): 509-519.

3. BASTOS RP, SOUZA DC. A prática do ensino estruturado no modelo TEACCH face à inclusão escolar do educando com TEA. Pesquisa e Prática em Educação Inclusiva, 2019; 2(4): 294-312.

4. BRANT DVS. Arte Circense como Recurso Terapêutico no Atendimento de Crianças com Transtorno do Espectro Autista. Revista Saúde e Educação, 2018; 3(2): 2-12.

5. CARDOSO NR, BLANCO MB. Terapia de integração sensorial e o transtorno do espectro autista: uma revisão sistemática de literatura. Revista Conhecimento Online, 2019; 1: 108-125.

6. CUNHA MCG, et al. Sistematização da Assistência de Enfermagem a criança autista na unidade hospitalar. Revista Interdisciplinar Pensamento Científico, 2019; 5(3): 2019.

7. DYKENS EM, et al. Reducing Distress in Mothers of Children With Autismand Other Disabilities: A Randomized Trial. Pediatrics, 2015; 134(2): 454-63.

8. FERNANDES CS, et al. Diagnóstico de autismo no século XXI: evolução dos domínios nas categorizações nosológicas. Psicologia USP, 2020; 31: e200027.

9. GOMES PTM, et al. Autism in Brazil: a systematic review of family challenges and coping strategies. Jornal de pediatria, 2015; 91: 111-121.

10. HADJKACEM I, et al. Fatores pré-natais, perinatais e pós-natais associados ao transtorno do espectro do autismo. Jornal de Pediatria, 2016; 92: 595-601.

11. HOFZMANN RR, et al. Experiência dos familiares no convívio de crianças com transtorno do espectro autista (TEA). Enfermagem em foco, 2019; 10(2): 64-69.

12. LIMA RC. Autismo e memória: neurociência e cognitivismo à luz da filosofia de Henri Bergson. Revista Latinoamericana de Psicopatologia Fundamental, 2020; 23: 745-768.

13. MANSUR OMFC, et al. Sinais de alerta para transtorno do espectro do autismo em crianças de 0 a 3 anos. Revista Científica da Faculdade de Medicina de Campos, 2017; 12(3).

14. MARQUES DF, BOSA CA. Protocolo de avaliação de crianças com autismo: evidências de validade de critério. Psicologia: Teoria e Pesquisa, 2015; 31(1): 43-51.

15. MARTINS JS, et al. Utilização de estímulos sensoriais táteis nas atividades pré-escolares de uma criança com TEA. Revista GepesVida, 2020; 6(14): 31-41.

16. MORAES LS, et al. Seletividade alimentar em crianças e adolescente com transtorno do espectro autista. Revista da Associação Brasileira de Nutrição-RASBRAN, 2021; $12(2): 42-58$.

17. OLIVEIRA CRA, SOUZA JC. Neurobiologia do autismo infantil. Pesquisa, sociedade e desenvolvimento, 2021; 10(1): e11910111495.

18. OLIVEIRA JJM, et al. Intervenção implementada pelos pais e empoderamento parental no transtorno do espectro autista. Psicologia Escolar e Educacional, 2020; 24: e218432.

19. PINTO AS, CONSTANTINIDIS TC. Revisão Integrativa sobre a Vivência de Mães de Crianças com Transtorno de Espectro Autista. Revista Psicologia e Saúde, 2020; 12 (2): 89-103.

20. PINTO RNM, et al. Autismo infantil: impacto do diagnóstico e repercussões nas relações familiares. Revista Gaúcha de enfermagem, 2016; 37(3): e61572.

21. POSAR A, VISCONTI P. Sensory abnormalities in children with autism spectrum disorder. Jornal de pediatria, 2018; 94(4): 342-350.

22. SANDIN S, et al. The Familial Risk of Autism. Journal of the American Medical Association, 2014; 311(17): 1770-1777.

23. SANTOS PA, et al. O impacto da implementação do Picture Exchange Communication System-PECS na compreensão de instruções em crianças com Transtorno do Espectro do Autismo. Communication Disorders, Audiology and Swallowing, 2021; 33 (2): e20200041.

24. SILVA ER, et al. Processamento sensorial: nova Dimensão na avaliação Das crianças com Transtorno do Espectro Autista. Revista Diálogos e Perspectivas em Educação Especial, 2016; 3(1): 62-76.

25. SIQUEIRA CC, et al. O cérebro autista: a biologia da mente e sua implicação no comprometimento social. Revista Transformar, 2016; 8(8): 221-237.

26. SOCIEDADE BRASILEIRA DE PEDIATRIA. Triagem precoce para Autismo/ Transtorno do Espectro Autista. 2017. Disponível em: https://www.sbp.com.br/fileadmin/user_upload/2017/04/19464b-DocCient-Autismo.pdf. Acessado em: 14 de maio de 2021.

27. STEYER S, et al. A Importância da Avaliação de Programas de Capacitação para Identificação dos Sinais Precoces do Transtorno do Espectro Autista-TEA. Trends in Psychology, 2018; 26(3): 395-1410.

28. TOMASI MC, et al. Sinestesia e o Transtorno do Espectro do Autismo (TEA). Revista de Atenção à Saúde, 2018; 16(55): 81-88.

29. VIANA KOFL, NASCIMENTO SS. Efeitos da intervenção precoce no desenvolvimento de uma criança com TEA: interface entre neurociências e educação. Perspectivas Online: Humanas \& Sociais Aplicadas, 2021; 11(30): 38-50.

30. VILANI MR, PORT IF. Neurociências e psicanálise: dialogando sobre o autismo. Estilos da Clínica, 2018; 23(1): $130-151$.

31. WENGER TL, et al. 22q11.2 duplication syndrome: elevated rate of autism spectrum disorder and need for medical screening. Molecular Autism, 2016; 7: 27

32. ZWAIGENBAUM L, et al. Early Identification of Autism Spectrum Disorder: Recommendations for Practice and Research. Pediatrics, 2015; 136: 10-40. 